\title{
Microalgae and Cyanobacteria: The Heroes of Growing Industrial Biotechnology Sectors
}

\section{Saha $\mathrm{SK}^{*}$ \\ Microalgal Biotechnologist, Limerick Institute of Technology Moylish Park, Ireland}

${ }^{*}$ Corresponding author: Sushanta Kumar Saha, Microalgal Biotechnologist, Shannon Applied Biotechnology Centre, Limerick Institute of Technology Moylish Park, Limerick, Ireland (ROI), Tel: +353 61 293536; E-mail: Sushanta.Saha@lit.ie

\section{Editorial \\ Volume 3 Issue 1}

Received Date: February 20, 2018

Published Date: March 01, 2018

DOI: $10.23880 /$ oajmb-16000123

\section{Editorial}

Microalgae and cyanobacteria are microscopic photosynthetic organisms similar to higher plants,found both in marine and freshwater environments, and are the pioneer of oxygenic environment that we are breathing today. These microorganisms possess immense morphological and biochemical diversity, which allow them to survive in varied and extreme environmental conditions. Several years of researching on them allowed to find their potential applications in production of fine chemicals for food and cosmetics applications; health supplements and therapeutic applications; bioenergy production; environmental management such as wastewater treatment, land reclamation and capture of green-house gases including $\mathrm{CO}_{2}$-fixation; in agriculture as atmospheric $\mathrm{N}_{2}$-fixer, phosphate solubiliser and source of various plant-growth promoting factors; etc. All these applications simply explore their photosynthetic machinery to capture the light energy and use minimal nutrients to produce the biomass with specific bioactive or other desired biomolecules as bio-factory (Figure 1).

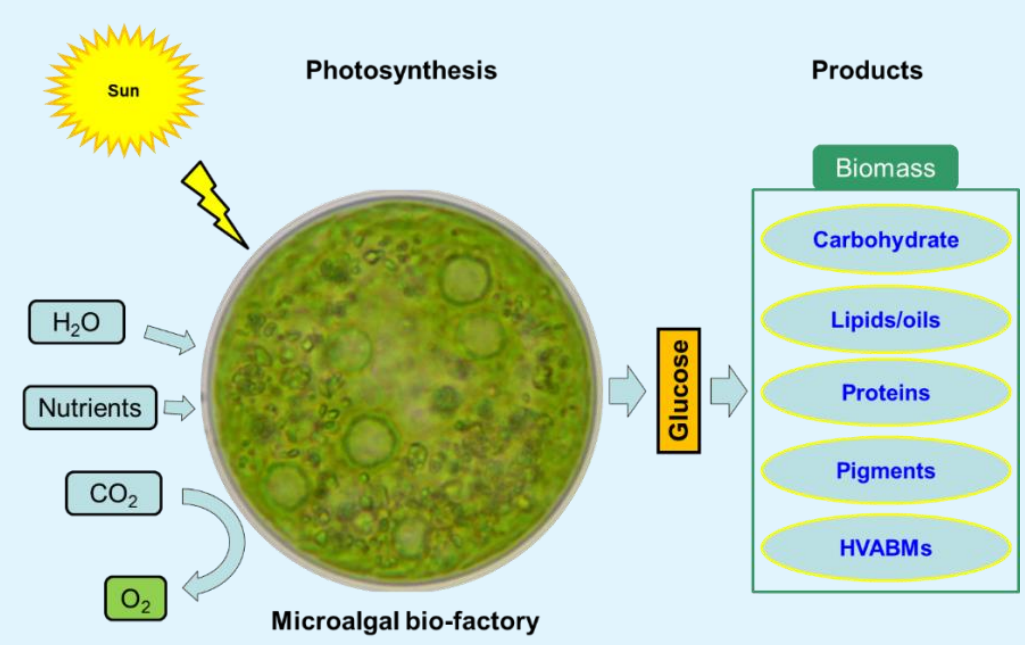

Figure 1: Schematic showing exploitation of microalgal or cyanobacterial photosynthetic machinery as bio-factory for biomass production for various biotechnological applications. 


\section{Open Access Journal of Microbiology \& Biotechnology}

Some very popular biomolecules of cyanobacteria and microalgae at present available in the market with huge demand are carotenoids (astaxanthin and $\beta$-carotene), phycobilipigments (phycocyanin and phycoerythrin), Omega-3 fatty acids (EPA and DHA). Whole biomass of Spirulinaplatensis,

Haematococcuspluvialis containing macromolecules (carbohydrate, lipids/oils, proteins), HVABMs (high value added biomolecules such as, anti-oxidants, anti-diabetics, etc.), essential minerals and vitamins for various food and cosmetics products and also as health supplement capsules, tablets etc. are in huge demand in the market. All these biotechnological development was achieved through optimisation of their sustainable cultivation technologies that does not depend on local weather nor need any arable lands, andmay be based on open raceway ponds, tubular or other closed photobioreactors.

Carotenoids are yellow, orange or red colour pigments used by microalgae and cyanobacteria as accessory photosynthetic pigments to trap light energy, and also to protect themselves from intense irradiation damage due to their anti-oxidative properties. Carotenoids especially astaxanthin and $\beta$-carotene from microalgae is very popular for various food including as supplements, functional ingredients and cosmetics applications. Microalga Haematococcuspluvialis, which is cultivated through two-stage cultivation: first green-phase for biomass production and second is the stress red-phase cultivation when each haematocysts accumulates astaxanthin and specific fatty acids occupying almost all cytoplasmic space of the round cells. Petrochemical based synthetic astaxanthin cost about $€ 1600$ per $\mathrm{kg}$, while natural astaxanthin from microalgae cost about $€ 5600$ per kg. Huge amounts of investments were made in USA, Israel, China, and also in various parts of Europe for the production of natural astaxanthin from microalga Haematococcuspluvialis using the robust and optimum cultivation conditions for meeting the demand for natural carotenoids for various biotechnological applications. Likewise, carotenoid $\beta$-carotene has commercially been produced from microalga Dunaliellasalina since 1966 in the USSR. The capability of commercial cultivation of $D$. salina is one of the oldest successful stories of marine biotechnology. However, the cost of $\beta$-carotene is not as high as astaxanthin and ranges €50-75 per $\mathrm{kg}$ and has much lower antioxidant activities compared to astaxanthin.

Phycobilipigments (PBPs) are a group of coloured protein-pigments composed of essential amino acids, commonly present in cyanobacteria and red microalgae as accessory photosynthetic pigments. PBPs are brilliant- coloured water-soluble pigments and are mainly in four colour shades: 1) allophycocyanin (APC, bluish green); 2) phycocyanin (PC, blue); 3) phycoerythrin (PE, purple) and 4) phycoerythrocyanin (PEC, orange)]. Both PC and PE have found various commercial applications, however, only PC have been commercially produced from cyanobacterium Spirulinaplatensis. Although, several cyanobacterial species were used for experimental identification and purification of phycoerythrin, have not been commercially used for PE production yet, which is a highly demanding biotechnology sector to be developed especially when there is a growing demand for natural food colourants. PC as natural food colourants have gained importance over synthetic colourants as they are natural, eco-friendly, non-toxic, non-carcinogenic and contributes to the essential amino acids pool as health benefit molecules. At present, PE are mostly produced commercially for diagnostic purposes and in diverse research applications from red microalga Porphyridiumcruentum. However, the production of foodgrade PE from this microalga is a major challenge due to the fact that this microalga produces huge amount of mucilaginous polysaccharides, which increases the cost of down-stream processing. Therefore, production of PE as a source of microalgal pink pigment is waiting for new strain and/or cheaper downstream technologies to be developed.

Omega-3 fatty acids are considered essential fatty acids as human beings need to depend for these fatty acids from their diet. Therefore there is a huge demand in health shops as supplements. Until recently, the source of these Omega-3 fatty acids (popularly known as EPA, DHA, etc.) were wild harvest 'Fish oil' and 'Krill oil'. However, nowadays "Vegetarian source" of these fatty acids are developing and less depending on wild harvest for these Omega-3 fatty acids. Namely, the heterotrophic microalga Crypthecodiniumcohnii; phototrophic diatom Phaeodactylumtricornutum and green microalga Nannochloropsis sp. are the heroes for omega-3 fatty acids production.

Apart from the above novel source of "health promoting" compounds, microalgae and cyanobacteria have been remained the prime focus of scientific research and development during the last decade for the development of the commercially viable biofuels including biodiesel, bioethanol and biogas production. More research is now focussed within the "Biorefinery concept" so that various microalgal commodities are obtained at acceptable 'cost of production' for each product including health supplements and/or biofuels. 


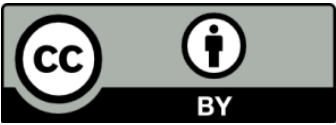

